\title{
6
}

\section{EL SECTOR EXPORTADOR ANTE LOS RIESGOS DE ABASTECIMIENTO}

El sector exterior ha contribuido a moderar el impacto económico de la pandemia sobre la economía y es también un elemento indispensable para asegurar una recuperación sostenible. La favorable inserción del sector exportador en cadenas globales de valor es clave para estos resultados. Sin embargo, esta inserción supone también una exposición a los riesgos que derivan de los episodios recurrentes de escasez que se han dado durante la pandemia y están marcando la recuperación de la actividad económica internacional.

En este artículo se realiza una aproximación al estudio de la exposición de los exportadores regulares de bienes a los riesgos de abastecimiento. Se parte del análisis general de la dependencia de nuestra economía de productos importados, que revela una concentración de importaciones potencialmente sensibles principalmente en China, Estados Unidos, algunos países del norte de África y otros de América Latina. El interés se centra, a continuación, en el sector exportador, estudiando su integración en cadenas globales de valor y la percepción de los exportadores regulares de bienes sobre el impacto de los episodios de escasez en su actividad. Se concluye con algunas recomendaciones orientadas a apoyar, mediante la política comercial, las medidas de diversificación y contención de riesgo que están ya adoptando los exportadores.

Palabras clave: comercio internacional, cadenas globales de valor, riesgo de abastecimiento, exportación, política comercial.

Clasificación JEL: F10, F13, F14.

\section{Introducción}

La inserción de la economía española en cadenas globales de valor alcanza niveles similares a los de las principales economías de nuestro entorno. Este resultado está muy vinculado a la inversión y al comercio internacionales y es indicativo de la capacidad

* Secretaría de Estado de Comercio.

Versión de febrero de 2022

https://doi.org/10.32796/bice.2022.3143.7352 de adaptación de las empresas españolas a los cambios que han tenido lugar, en las últimas décadas, en el entorno económico internacional.

Este favorable posicionamiento ha posibilitado que las empresas españolas continuaran accediendo a los mercados exteriores en los momentos en los que, como resultado de las medidas de contención de la pandemia y de la adaptación del comportamiento de los agentes económicos al nuevo entorno, la demanda $\square$ 
interna era más débil. El sector exterior ha contribuido por esta vía a moderar el impacto negativo de la pandemia sobre la economía y el empleo. También ha facilitado el acceso a los medicamentos y productos sanitarios necesarios para la lucha contra la COVID-19. Así, el sector exterior también ha sido clave para atenuar los efectos de la pandemia en la salud de la población.

La crisis sanitaria global está impulsando una reorganización de las cadenas globales de valor que puede ser una fuente tanto de oportunidades como de riesgos. El Plan de Acción para la Internacionalización de la Economía Española, 2020-2021 (Ministerio de Industria Comercio y Turismo, 2021a), recoge actuaciones orientadas tanto a mejorar el aprovechamiento de las oportunidades que puedan surgir como consecuencia de la reorganización de las cadenas globales de valor como al refuerzo de su seguridad, mediante el impulso a la creación de un marco estable, basado en reglas, la diversificación de la oferta, la apertura de mercados o el desarrollo de marcos cooperativos que faciliten el acceso a productos críticos (Subdirección General de Estrategia de Internacionalización, 2021).

Los episodios de escasez observados, tanto en las fases más agudas de la crisis sanitaria como en la de recuperación de la actividad económica, confirman la necesidad de impulsar este tipo de actuaciones. Para garantizar su eficacia es necesario estudiar en profundidad el papel que desempeñan las cadenas globales de valor en nuestra economía y las oportunidades y riesgos que llevan asociados.

El objetivo de este artículo es contribuir a este estudio, ofreciendo un análisis preliminar referido al ámbito de la exportación de bienes. Para ello, se parte del análisis general sobre las posibles dependencias de la economía española desde la vertiente importadora para, a continuación, estudiar la inserción de las empresas exportadoras en cadenas globales de valor, su exposición ante los riesgos que se han materializado en los últimos meses en el ámbito de los suministros internacionales, y su capacidad de adaptación a este escenario.

De acuerdo con este objetivo, en el siguiente epígrafe se presentan los resultados de una aproximación cuantitativa a las importaciones para las que la economía española depende de un número reducido de proveedores extracomunitarios. En el tercer epígrafe se analizan la integración de la exportación española en las cadenas globales de valor y la visión de los exportadores regulares de bienes sobre esta integración. Así, se estudia tanto el grado en que consideran que su actividad depende de productos importados como su apreciación sobre la medida en que los productos que exportan se incorporan a exportaciones de terceros países. El epígrafe cuarto se centra en estudiar la percepción de estos exportadores sobre los problemas de abastecimiento registrados en los últimos meses y en analizar el tipo de medidas que están adoptando para gestionar este tipo de riesgos. En un último epígrafe se destacan las conclusiones más relevantes y sus implicaciones para las políticas de apoyo a la internacionalización.

\section{Análisis de la dependencia de productos importados}

En este epígrafe se ofrece una primera aproximación al análisis de dependencia que presenta la economía española para la importación de determinados productos de un número reducido de países proveedores no pertenecientes a la Unión Europea. Los resultados $\triangleright$ 
preliminares revelan una concentración de riesgos de vulnerabilidad en importaciones procedentes de China y en sectores como los de minerales y combustibles, maquinaria o productos químicos. Estos resultados presentan algunos rasgos comunes a los identificados para el conjunto de la Unión Europea, aunque el análisis revela también algunas diferencias destacables.

Los recurrentes episodios de escasez registrados tanto en las primeras fases de la pandemia como en la etapa de fuerte recuperación de la demanda, iniciada a mediados de 2021, han generado un interés creciente por analizar las vulnerabilidades de la economía ante posibles restricciones de la oferta en otros países. En Recuperación económica y problemas de abastecimiento: el papel de los fletes se ofrece al lector interesado un análisis detallado de las diversas causas que han incidido en estos episodios de escasez, profundizando en el papel del transporte marítimo de mercancías, de sus posibles efectos y de las actuaciones a desarrollar, tanto desde el ámbito empresarial como desde el de las políticas comerciales, para afrontarlos (Subdirección General de Estudios y Evaluación de Instrumentos de Política Comercial, 2021).

Las restricciones, asociadas a cuestiones sanitarias, geopolíticas, climáticas u otras posibles causas, pueden manifestarse en dificultades de acceso a determinados productos importados, incrementos de sus precios o retrasos en su entrega. Estas dificultades tendrán mayor repercusión económica si los productos afectados son difícilmente sustituibles, ya sea mediante su importación desde otros países o regiones, ya sea mediante la producción nacional, o si afectan a áreas o actividades que puedan considerarse estratégicas para el desarrollo del país.
La metodología aplicada en este epígrafe para identificar las importaciones para las que la dependencia geográfica es relevante es una adaptación de la desarrollada por la Comisión Europea para el análisis de las dependencias estratégicas de la Unión Europea (Comisión Europea, 2021) y se describe en el Recuadro 1. Consiste básicamente en la combinación de tres indicadores de la dependencia de la economía española respecto de cada producto importado: la concentración de las importaciones, que mide su concentración geográfica; la tasa de cobertura, que está relacionada con la posibilidad de sustituir estas importaciones por producción nacional; y la dependencia extracomunitaria, que muestra el grado en que estas importaciones proceden de terceros países y suponen, por tanto, un nivel de riesgo mayor que el derivado de las procedentes del mercado interior europeo.

El análisis efectuado con datos referidos al año 2019 que, por tanto, no incorpora las distorsiones derivadas de la pandemia COVID-19, permite identificar un total de 347 productos en los que la economía española presenta, de acuerdo con los indicadores definidos, una dependencia elevada de países extracomunitarios. En valor, estos productos representaron en torno al $7,0 \%$ del total de las importaciones españolas de bienes en 2019. Este porcentaje es similar al $6 \%$ observado en el conjunto de la Unión Europea, aunque, en este caso, la dependencia se concentra en tan solo 137 productos (Comisión Europea, 2021).

Con relación a su origen geográfico, destaca la dependencia de productos importados desde China, que es el principal proveedor de 158 de los productos identificados y el origen del $24 \%$ del valor de estas importaciones (Gráfico 1). Argelia (12\%) y Marruecos (11\%), en el norte de África, y, en menor medida, $\square$ 
RECUADRO 1

METODOLOGÍA DE ANÁLISIS DE RIESGO EN EL SUMINISTRO DE IMPORTACIONES

Se estudia el país de origen de los grupos de productos importados por España en 2019, clasificados en códigos de la nomenclatura combinada a seis dígitos (5.294 productos o subpartidas). Para cada uno de los productos se elaboran los siguientes indicadores de riesgo de suministro:

- Concentración de las importaciones. Se utiliza el índice de Herfindahl-Hirschman (HHI) que mide la concentración a partir de las cuotas de mercados de los países proveedores de España. Se considera un posible riesgo cuando $\mathrm{HHI}>0,4$, lo que implica que un elevado porcentaje de las importaciones se concentra, por lo general, en un máximo de dos o tres países.

- Tasa de cobertura. Se requiere que el saldo de comercio internacional del producto para España sea negativo (tasa de cobertura < 100\%). Si el saldo fuera positivo, ante problemas de abastecimiento cabría sustituir importaciones por producción nacional destinada a la exportación. Se trata de una mera aproximación, ya que cada subpartida, definida a seis dígitos, engloba en realidad productos diversos, que pueden tener características y usos diferentes.

- Dependencia extracomunitaria. Mide la proporción de importaciones extracomunitarias sobre las importaciones totales. Conforme a la metodología aplicada por la Comisión Europea, si más del $50 \%$ de las importaciones proceden de fuera de la Unión Europea (UE), se considera que existe un riesgo potencial. La dependencia de proveedores de la UE no conlleva igual riesgo, dadas las garantías que ofrece el mercado interior.

Si un producto supera los umbrales establecidos para cada uno de estos indicadores y, además, el valor de su importación es superior al millón de euros, se considera que existe dependencia de su importación.

Para facilitar el análisis, se han definido ocho grupos de productos, agregando capítulos de la nomenclatura combinada:

\begin{tabular}{|l|c|}
\hline \multicolumn{1}{|c|}{ Grupos } & Capítulos \\
\hline Agroalimentación & $1-24$ \\
\hline Minerales y combustibles & $25-27$ \\
\hline Productos químicos (incluidos medicamentos y plásticos) & $28-40$ \\
\hline Madera, papel, piel, textiles y manufacturas de consumo & $41-71$ y $94-97$ \\
\hline Metales y sus manufacturas & $72-83$ \\
\hline Maquinaria & $84-85$ \\
\hline Material de transporte & $86-89$ \\
\hline Instrumentos y aparatos de precisión & $90-92$ \\
\hline
\end{tabular}

Fuente: Elaboración propia, a partir de Comisión Europea, 2021.

Estados Unidos (8\%), Brasil (5\%) y Argentina (3\%), en América, son también los principales proveedores de un notable porcentaje de los productos importados en los que se detecta dependencia.

Estos datos presentan algunas diferencias significativas con los relativos al conjunto de la Unión Europea (Gráfico 2), que muestran una concentración más acusada en China (que absorbe el $52 \%$ del valor de las importaciones para las que se detecta dependencia) y otras economías asiáticas, como Vietnam (11\%), Corea del Sur (4\%) o Singapur (4\%). Entre los países latinoamericanos también destaca Brasil $(5 \%)$.

Desde la perspectiva sectorial (Gráfico 3), el mayor número de productos importados en los que se identifica dependencia pertenece al $D$ 


\section{EL SECTOR EXPORTADOR ANTE LOS RIESGOS DE ABASTECIMIENTO}

GRÁFICO 1

IMPORTACIONES DE ESPAÑA. ORIGEN GEOGRÁFICO DE LOS PRODUCTOS CON DEPENDENCIA

(Porcentaje del valor)

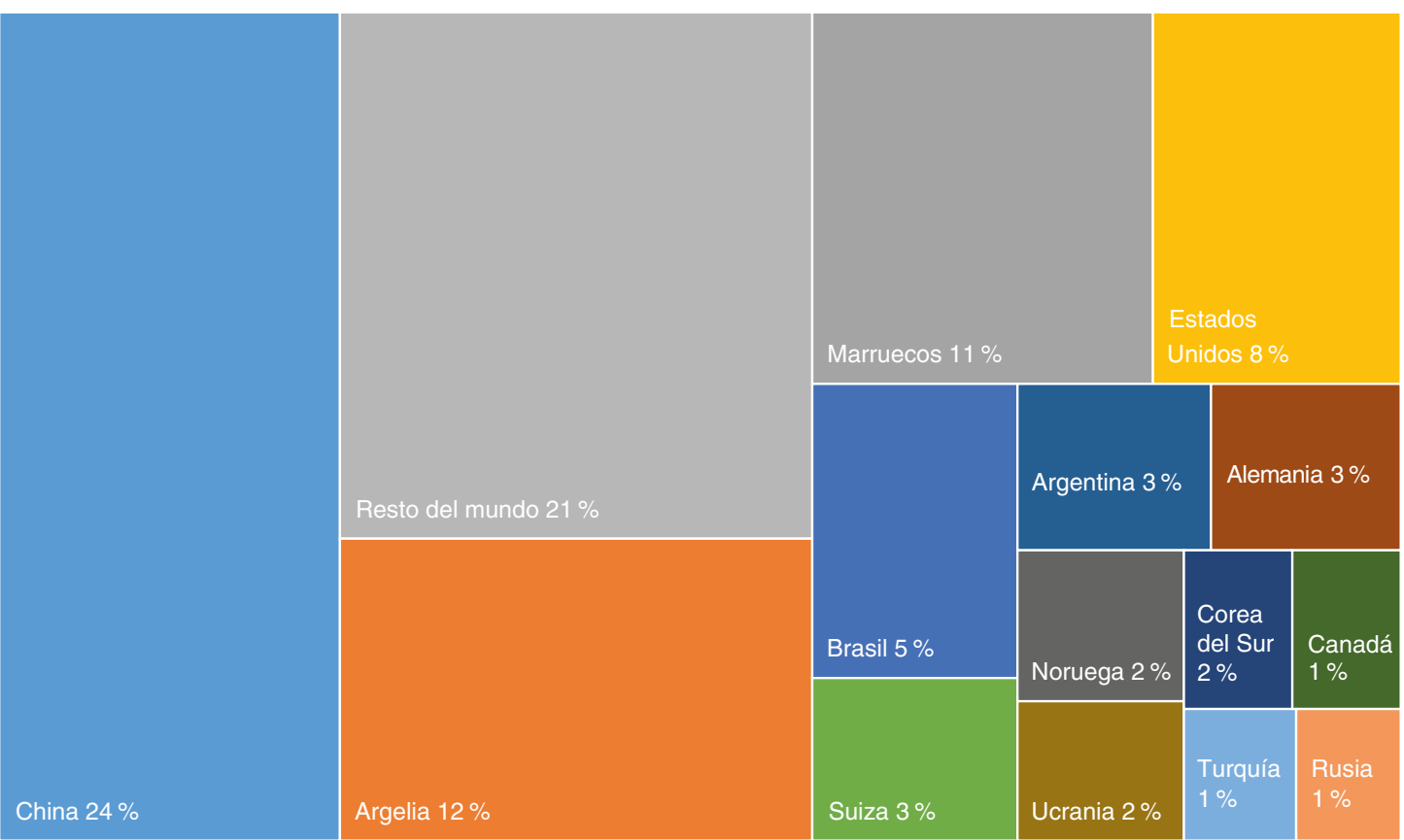

Fuente: Web DataComex y elaboración propia.

GRÁFICO 2

IMPORTACIONES DE LA UE. ORIGEN GEOGRÁFICO DE LOS PRODUCTOS CON DEPENDENCIA

(Porcentaje del valor)

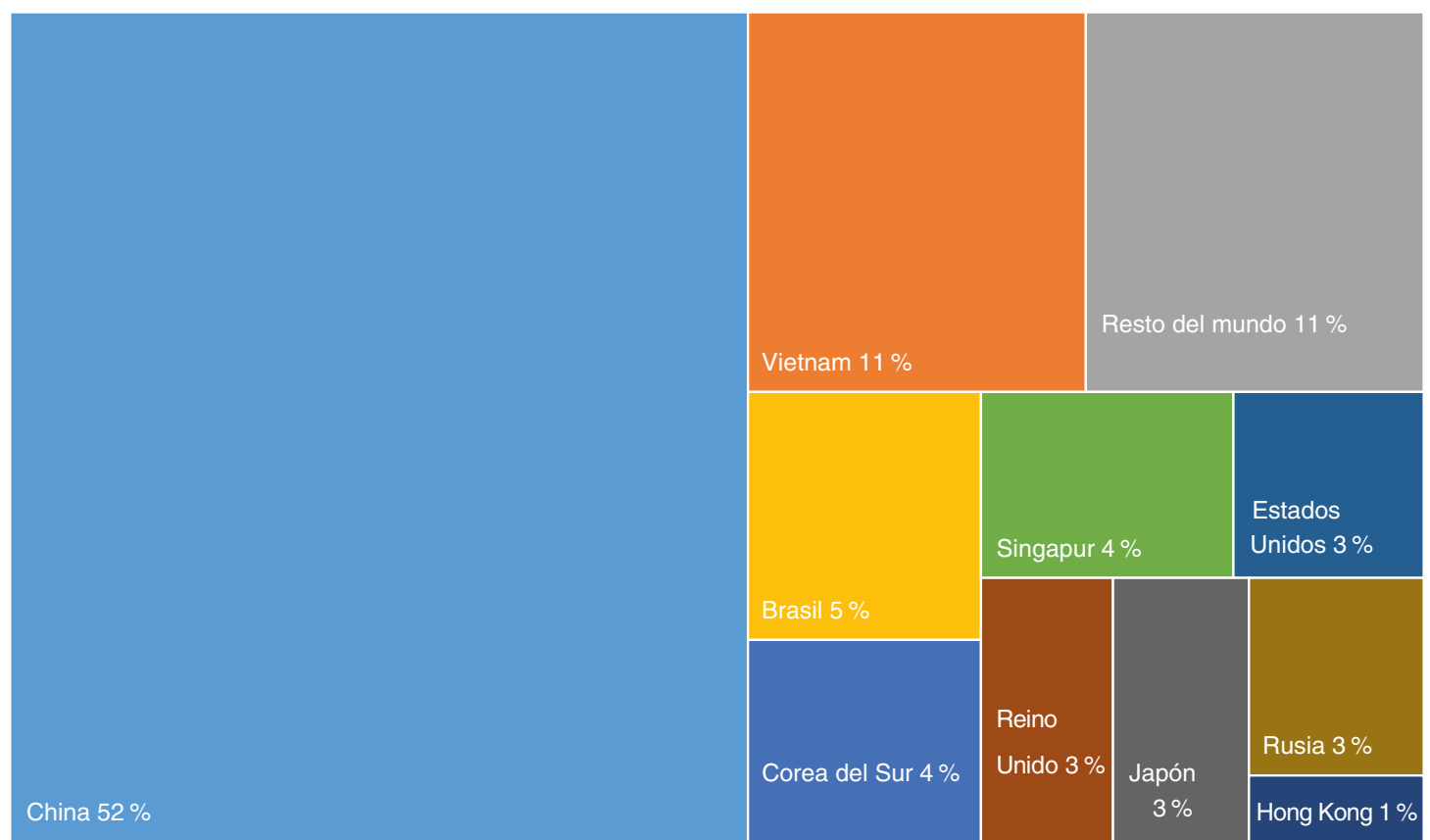

Fuente: Comisión Europea, 2021. 
Subdirección General de Estudios y Evaluación de Instrumentos de Política Comercial

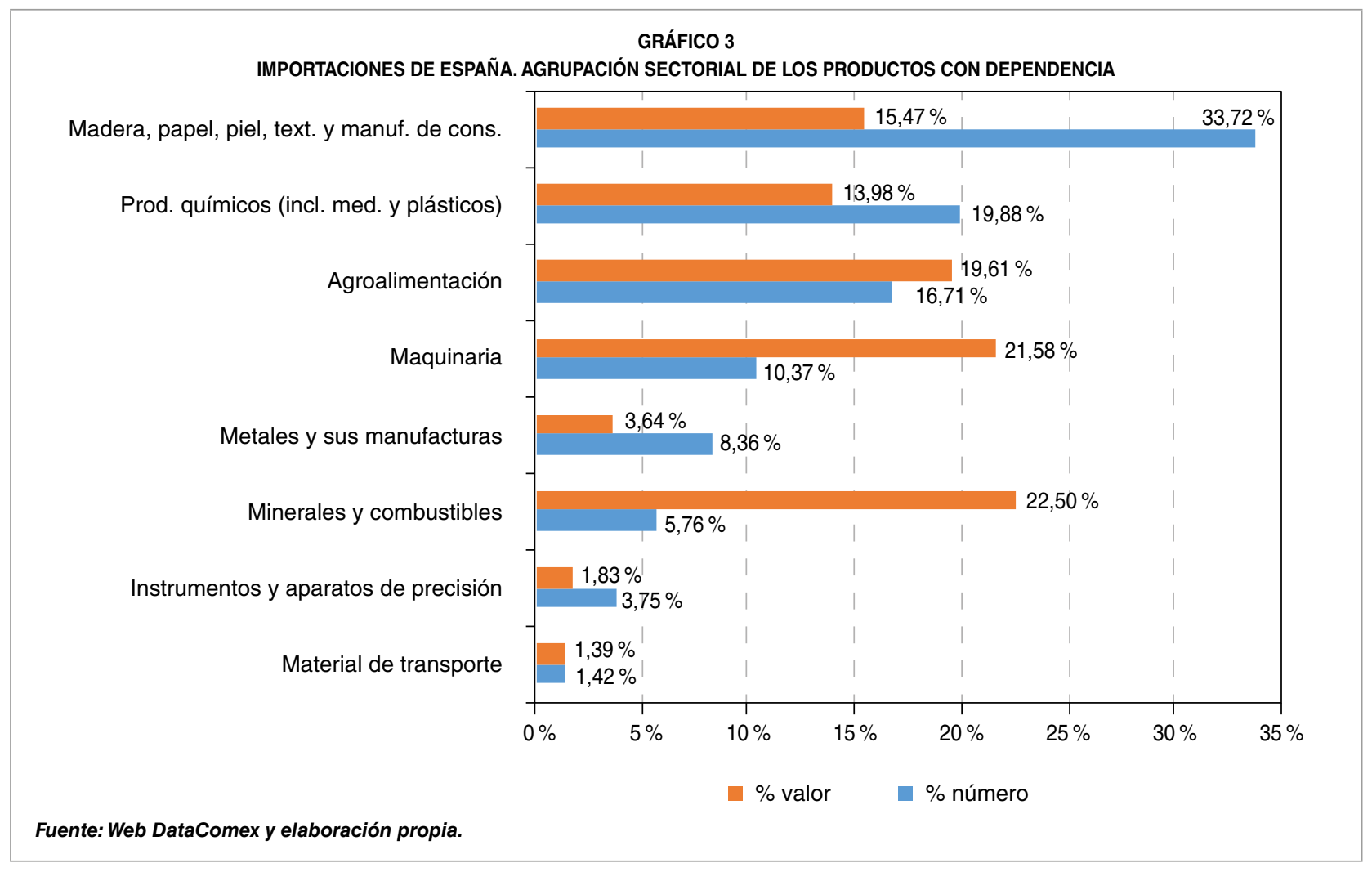

grupo de madera, papel, piel, textil y manufacturas de consumo, que incluye 117 productos (el $34 \%$ de los identificados). Los siguientes grupos con mayor presencia de productos con dependencia son los de productos químicos, donde se incluyen medicamentos y plásticos (20\%), y agroalimentación (17\%). Si el análisis se efectúa en términos del valor de las importaciones, cobran mayor importancia los grupos de minerales y combustibles (22\%) y maquinaria $(22 \%)$.

Estos resultados deben interpretarse de acuerdo con su naturaleza de exploración preliminar. El análisis efectuado permite identificar los productos en los que España presenta una dependencia concentrada en pocos mercados de origen. Este análisis inicial podría perfeccionarse desarrollando, por ejemplo, indicadores complementarios de sustituibilidad que reflejen la existencia de proveedores alternativos (intracomunitarios o extracomunitarios) a los actuales, o ponderando el resultado en función de que los países frente a los que se detecta dependencia presenten distintos niveles de riesgo geopolítico, estén dotados o no de acuerdos comerciales con la Unión Europea, etcétera.

En todo caso, más allá del perfeccionamiento de los indicadores de dependencia, se requiere un estudio producto a producto para determinar su relevancia estratégica para la economía española. En este sentido, cabría considerar que la mayor parte de las manufacturas de consumo, así como de los productos textiles, de piel o madera, y, en cierta medida, de los productos agroalimentarios, tienen una menor importancia estratégica, ya que, en muchos casos, cuentan con sustitutivos adecuados que, llegado el caso, pueden reemplazarlos sin generar un elevado impacto en la $D$ 
economía. Bajo este supuesto, podría concluirse que los principales riesgos de vulnerabilidad para la economía española se concentran en los sectores de minerales y combustibles, maquinaria y productos químicos.

El análisis realizado por la Comisión Europea identifica dependencias de la importación también en estos ámbitos. Así, se identifican diversas materias primas minerales, aleaciones de metales o productos químicos que son clave para el ecosistema energético. Se apunta también a la importancia de productos químicos que son principios activos necesarios para la producción de fármacos, así como a la relevancia, tanto de materias primas minerales como de maquinaria y componentes, para la transición verde y digital (Comisión Europea, 2021).

\section{Integración del sector exportador en cadenas globales de valor}

El elevado grado de integración del sector exportador en cadenas globales de valor revela la capacidad que ha tenido la economía española de aprovechar las oportunidades que ha ofrecido el proceso de globalización económica observado en las últimas décadas. No obstante, en un contexto de reestructuración de las relaciones económicas internacionales, marcado por tensiones geopolíticas, y de episodios de escasez y riesgo de desabastecimiento, la supervisión de las cadenas globales de valor en que se integra el sector exportador y el análisis de su resiliencia cobran especial relevancia.

Como se ha señalado, la economía española presenta un grado de integración en cadenas globales de valor equiparable al de las principales economías de nuestro entorno. Así, nuestras exportaciones dependen en un grado similar de bienes o servicios importados (integración hacia atrás o backward) y se utilizan en un porcentaje algo inferior para producir bienes o servicios en los países de destino que, a su vez, son objeto de exportación (integración hacia delante o forward).

En concreto, con datos de 2018, las exportaciones españolas incorporan un $23,8 \%$ de valor añadido bruto procedente de bienes o servicios importados. Este porcentaje es semejante al de las principales economías de la Unión Europea (Alemania, Francia e Italia) y superior al observado en Reino Unido (Gráfico 4). A su vez, el $18,6 \%$ del valor añadido bruto exportado por España se emplea, en los países de destino, para la producción de bienes o servicios que se destinan, a su vez, a la exportación. Este porcentaje es algo inferior al observado en Italia $(19,9 \%)$ y se sitúa claramente por debajo del registrado en Alemania $(23,4 \%)$, Francia (22,0\%) o Reino Unido (24,0\%), lo que podría indicar que aún existe un margen de mejora en este ámbito.

La percepción de los exportadores regulares de bienes sobre su inserción en cadenas globales de valor apunta en la misma línea de estos resultados, ya que sugiere un mayor grado de integración hacia atrás y una participación más moderada hacia adelante (Gráfico 5 ). Así, en torno al 55,9\% de los exportadores regulares son conscientes de que su producción depende bastante $(34,3 \%)$ o moderadamente $(21,6 \%)$ de bienes o servicios importados. La percepción sobre la incorporación de las exportaciones a la producción de bienes o servicios que, a su vez, serán exportados es menos elevada. Solo el $27,6 \%$ de los exportadores regulares considera que una parte significativa de los bienes o servicios que exporta su empresa se destinan a este fin. 
Subdirección General de Estudios y Evaluación de Instrumentos de Política Comercial
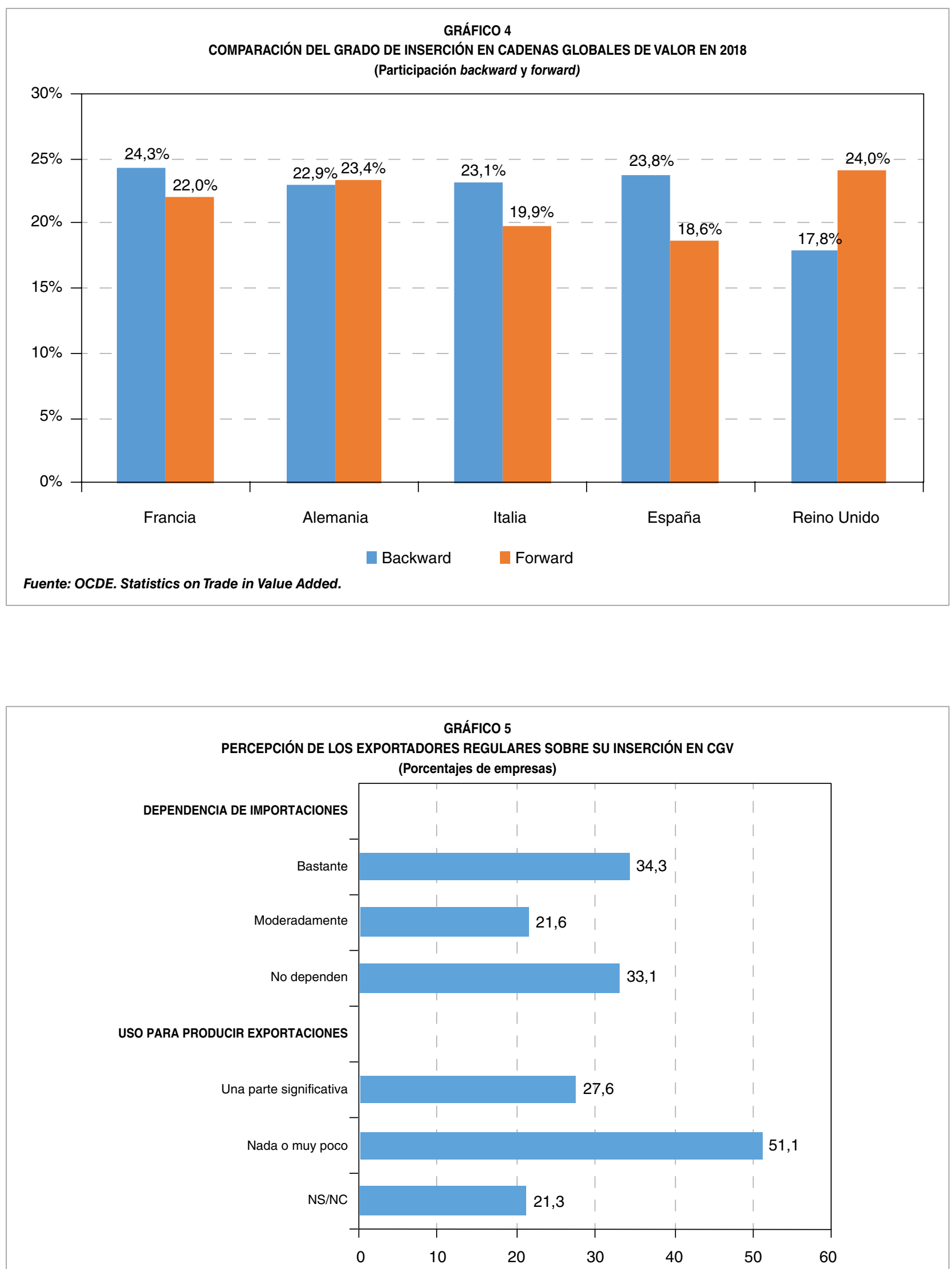

Fuente: Encuesta de Coyuntura de la Exportación, III trimestre de 2021. 
Los indicadores analizados apuntan, por lo tanto, a una elevada integración hacia atrás (backward) en las cadenas globales de valor y a una integración hacia adelante (forward), donde parece existir un margen de mejora.

Así, la elevación de la participación hacia adelante facilitaría un mayor aprovechamiento del dinamismo del comercio internacional, no solo a través del crecimiento directo de la exportación española hacia los mercados de mayor crecimiento, sino también a través del crecimiento de las exportaciones de terceros países que incorporen valor añadido de origen español.

La elevada integración hacia atrás es, por otro lado, una fortaleza de nuestro sector exportador, ya que le permite, a través de la importación, acceder a los mejores insumos y tecnologías de los mercados mundiales, con ganancias de eficiencia, reducciones de coste $y$, en última instancia, mejoras de competitividad. No obstante, en el contexto actual de episodios recurrentes de escasez y en el horizonte de medio plazo, en el que se espera una reorganización de las cadenas globales de valor, es también una fuente de riesgo potencial que es preciso examinar.

\section{Los problemas de abastecimiento y la exportación}

Los problemas de abastecimiento repercuten en el sector exportador. Especialmente desde mediados de 2021, las empresas exportadoras de bienes se enfrentan a dificultades de aprovisionamiento, derivadas principalmente de los mercados internacionales, que limitan su capacidad de producir o exportar. A corto plazo, están recurriendo a la diversificación de proveedores y de medios de transporte para afrontar estos cuellos de botella, aunque algunos exportadores se han visto obligados a reducir su producción o exportación. A medio plazo, las empresas exportadoras prevén aplicar un abanico más amplio de medidas, no exentas de coste, que incluyen la diversificación de proveedores, el acortamiento y supervisión de sus cadenas de suministro o el aumento del stock de existencias.

El análisis de la repercusión de los problemas de abastecimiento en el sector exportador tiene especial interés ya que el dinamismo de este sector es un apoyo clave para la recuperación del conjunto de la economía española y para su crecimiento sostenible. En este epígrafe se estudia la forma en que están percibiendo las empresas que exportan regularmente bienes los actuales problemas de abastecimiento y las medidas que están adoptando para paliarlos. El análisis se efectúa a partir de los resultados del módulo sobre problemas de abastecimiento incluido en la Encuesta de Coyuntura de la Exportación (ECE) del IV trimestre de 2021 (Ministerio de Industria, Comercio y Turismo, 2021b). En el Recuadro 2 se resume la ficha técnica de esta encuesta. Las preguntas efectuadas en el módulo admiten, en algunos casos, respuesta múltiple. Las contestaciones en estos casos se han reponderado, de forma que las distintas opciones sumen el $100 \%$ y el valor obtenido pueda interpretarse como una medida de la importancia relativa que el sector de exportadores regulares de bienes atribuye, en su conjunto, a cada alternativa.

Los exportadores regulares sitúan, mayoritariamente, el inicio de los problemas de abastecimiento en el año 2021 y, en particular, a partir del segundo trimestre del año (Gráfico 6). Este perfil coincide con el momento de recuperación y fortalecimiento de la actividad económica. 
RECUADRO 2

FICHA TÉCNICA DE LA ENCUESTA DE COYUNTURA DE LA EXPORTACIÓN

Ámbito: nacional.

Universo: empresas que realizan, de forma continua, operaciones de exportación de mercancías superiores a 30.000 euros anuales en todos y cada uno de los cuatro años del periodo 2016-19 (en total, 25.889 empresas).

Muestra: 1.900 empresas.

Tipo de muestreo: aleatorio estratificado. Las variables de estratificación son el sector principal de exportación y el tamaño de la empresa (medido por el valor anual medio de las exportaciones de la empresa en el periodo 2016-19). El número total de estratos considerados es de 36.

Afijación muestral: mixta (proporcional al número de empresas y al valor de las exportaciones por estrato).

Selección de la muestra: aleatoria sin reposición del número de unidades (empresas) requeridas en cada estrato.

Informantes: responsables de exportación de las empresas seleccionadas.

Entrevista: autocumplimentación por CAWI (web) y entrevista telefónica, asistida por ordenador, con una duración media de doce minutos.

Fecha de recogida: del 15 de noviembre al 15 de diciembre de 2021.

Fuente: Ministerio de Industria, Comercio y Turismo, 2021 b.

Los problemas de abastecimiento que perciben los exportadores regulares se relacionan principalmente con el ámbito logístico, señalando dificultades como retrasos en la recepción de los pedidos (con una importancia relativa del $30,4 \%)$ o elevación de los costes de transporte $(29,7 \%)$. La importancia relativa que los exportadores atribuyen a problemas para encontrar el producto deseado $(16,8 \%)$ o al incremento de sus precios $(23,1 \%)$ es más reducida.

Además, los exportadores regulares estiman que estos problemas no se van a resolver de forma inmediata. Solo el $15,1 \%$ confía en que duren menos de seis meses, mientras que la opinión del $62,1 \%$ de los exportadores es que persistirán entre seis y doce meses y un $22,8 \%$ consideran que tendrán mayor duración.

Los problemas de abastecimiento son sectorialmente generalizados y están vinculados a los mercados internacionales. En concreto, las dificultades de suministro están limitando la producción o la exportación del $60,2 \%$ de los exportadores regulares (Cuadro 1). Estos problemas están presentes en todos los sectores de exportación, aunque con distinta intensidad. Así, en la exportación de materias primas solo el 32,4\% de los exportadores perciben problemas que afectan a sus capacidades de producir o exportar, mientras que en el de bienes de equipo, estas limitaciones afectan al 69,8\% de las empresas.

Globalmente, el $92,1 \%$ de los exportadores regulares que perciben problemas de suministro los relaciona con los mercados internacionales, bien de forma exclusiva $(40,5 \%)$ o en conjunción con dificultades encontradas también en el mercado nacional $(51,6 \%)$. En todos los sectores de exportación se observa esta fuerte vinculación entre los problemas de suministro detectados y los mercados internacionales. En el caso de los productos energéticos, el $100 \%$ de los exportadores que perciben dificultades de abastecimiento las atribuye a los mercados $D$ 


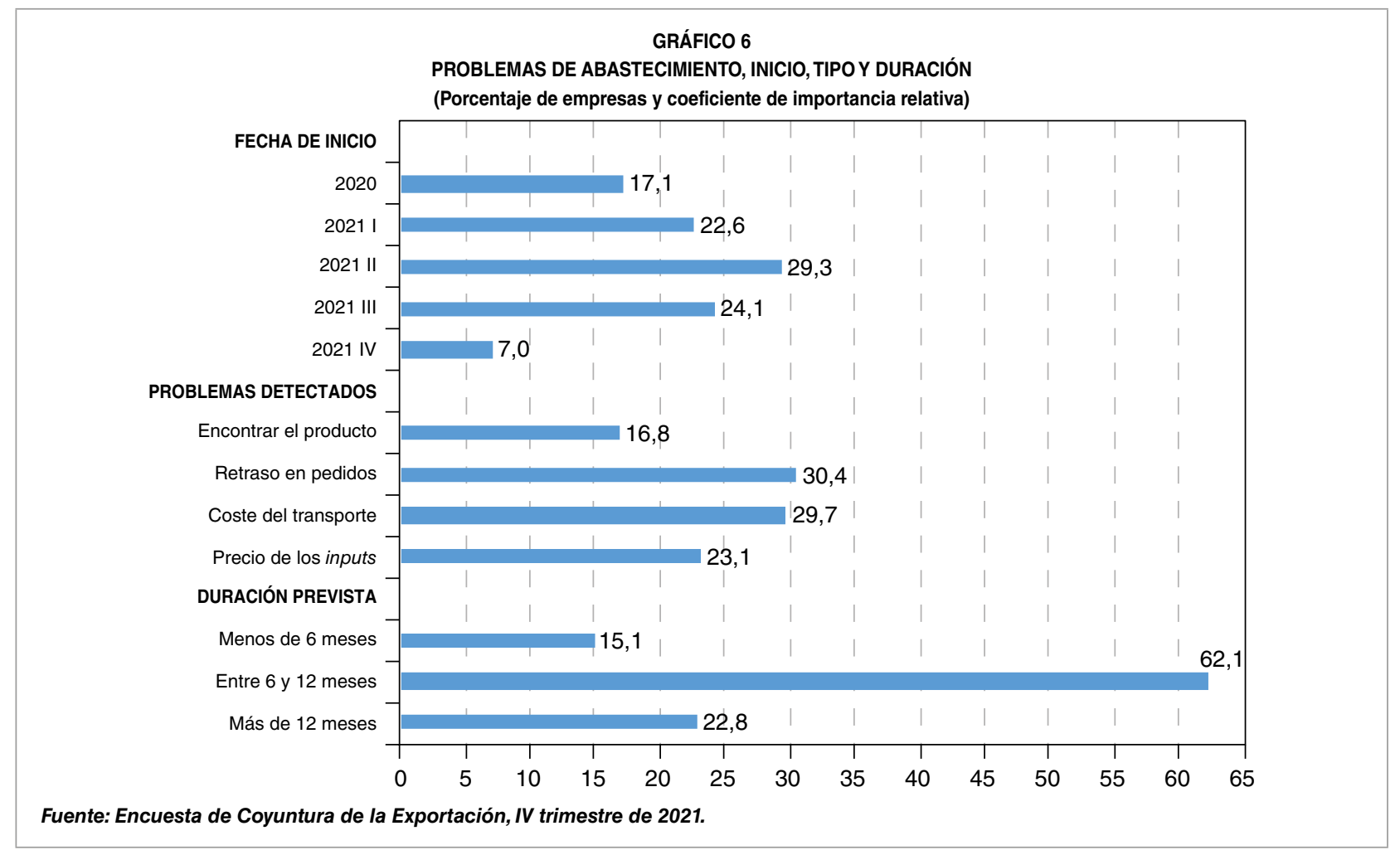

internacionales, ya sea en exclusiva $(50,0 \%)$ o en combinación con dificultades en el mercado doméstico (50,0\%). En el caso del automóvil, el $99,8 \%$ de las empresas que ha experimentado dificultades de suministro las relaciona con los mercados internacionales, predominando las que solo observan problemas en estos mercados $(62,3 \%)$ sobre las que detectan problemas tanto en mercados internacionales como en el nacional (37,5\%). En el sector de la alimentación, bebidas y tabaco es donde se aprecia una mayor incidencia de las dificultades de suministro de productos adquiridos en el mercado nacional $(25,4 \%)$. Aunque el porcentaje de empresas de este sector que encuentra dificultades en los mercados internancionales $(31,1 \%)$ o en ambos mercados (43,5\%) es superior.

La mayor parte de los exportadores regulares que experimentan problemas de abastecimiento están adoptando medidas para paliarlos. En general, estas medidas se orientan a la búsqueda de productos, proveedores o países de origen alternativos, así como a cambios en los medios de transporte. En otros casos, las dificultades de suministro están siendo compensadas con reducciones de la producción o de las exportaciones.

En concreto, el $66,8 \%$ de los exportadores regulares ha adoptado medidas para moderar el impacto de los problemas de suministro (Gráfico 7). Estos exportadores que han adoptado una o varias medidas de atenuación del impacto atribuyen un peso del $42,7 \%$ a las orientadas a modificar productos, proveedores - países en los que se abastecen, mientras que la búsqueda de medios de transporte alternativos tiene una importancia relativa del $23,4 \%$. Este porcentaje, junto con el atribuido por los exportadores regulares a los problemas de retraso en el suministro y coste del transporte, es indicativo de la importancia que están teniendo los trastornos en la cadena logística en los episodios de escasez. 
Subdirección General de Estudios y Evaluación de Instrumentos de Política Comercial

CUADRO 1

EXPORTADORES REGULARES QUE EXPERIMENTAN PROBLEMAS DE ABASTECIMIENTOY ORIGEN DE LOS PROBLEMAS (Porcentaje de empresas)

\begin{tabular}{|c|c|c|c|c|c|c|}
\hline \multirow[t]{2}{*}{ Sectores } & \multicolumn{3}{|c|}{$\begin{array}{c}\text { Experimenta problemas de } \\
\text { abastecimiento que limitan sus } \\
\text { posibilidades de producción y } \\
\text { exportación }\end{array}$} & \multicolumn{3}{|c|}{$\begin{array}{l}\text { Estos problemas proceden de } \\
\text { suministros adquiridos en el } \\
\text { mercado }\end{array}$} \\
\hline & Sí & No & NS/NC & Nacional & Internacional & Ambos \\
\hline Alimentación, bebidas y tabaco & 51,1 & 43,9 & 4,9 & 25,4 & 31,1 & 43,5 \\
\hline Productos energéticos & 43,2 & 56,8 & 0,0 & 0,0 & 50,0 & 50,0 \\
\hline Materias primas & 32,4 & 67,6 & 0,0 & 10,1 & 49,9 & 40,0 \\
\hline Semimanufacturas no químicas & 52,1 & 37,1 & 10,8 & 12,7 & 27,1 & 60,2 \\
\hline Productos químicos & 61,8 & 31,5 & 6,8 & 2,2 & 37,4 & 60,4 \\
\hline Bienes de equipo & 69,8 & 26,1 & 4,1 & 6,0 & 36,2 & 57,8 \\
\hline Sector del automóvil & 46,3 & 48,5 & 5,2 & 0,2 & 62,3 & 37,5 \\
\hline Bienes de consumo duradero & 63,3 & 30,9 & 5,7 & 11,2 & 28,2 & 60,6 \\
\hline Manufacturas de consumo & 60,2 & 34,0 & 5,9 & 3,1 & 50,9 & 46,0 \\
\hline Total & 56,0 & 38,7 & 5,3 & 7,9 & 40,5 & 51,6 \\
\hline
\end{tabular}

El análisis sectorial revela que el cambio de producto, proveedor o país de origen es la medida a la que las empresas exportadoras que han adoptado algún tipo de medida atribuyen mayor importancia relativa en todos los sectores, salvo en el del automóvil y en el de bienes de $\triangleright$

\section{GRÁFICO 7}

MEDIDAS ADOPTADAS Y PREVISTAS POR LAS EMPRESAS

(Porcentaje de empresas y coeficiente de importancia relativa)

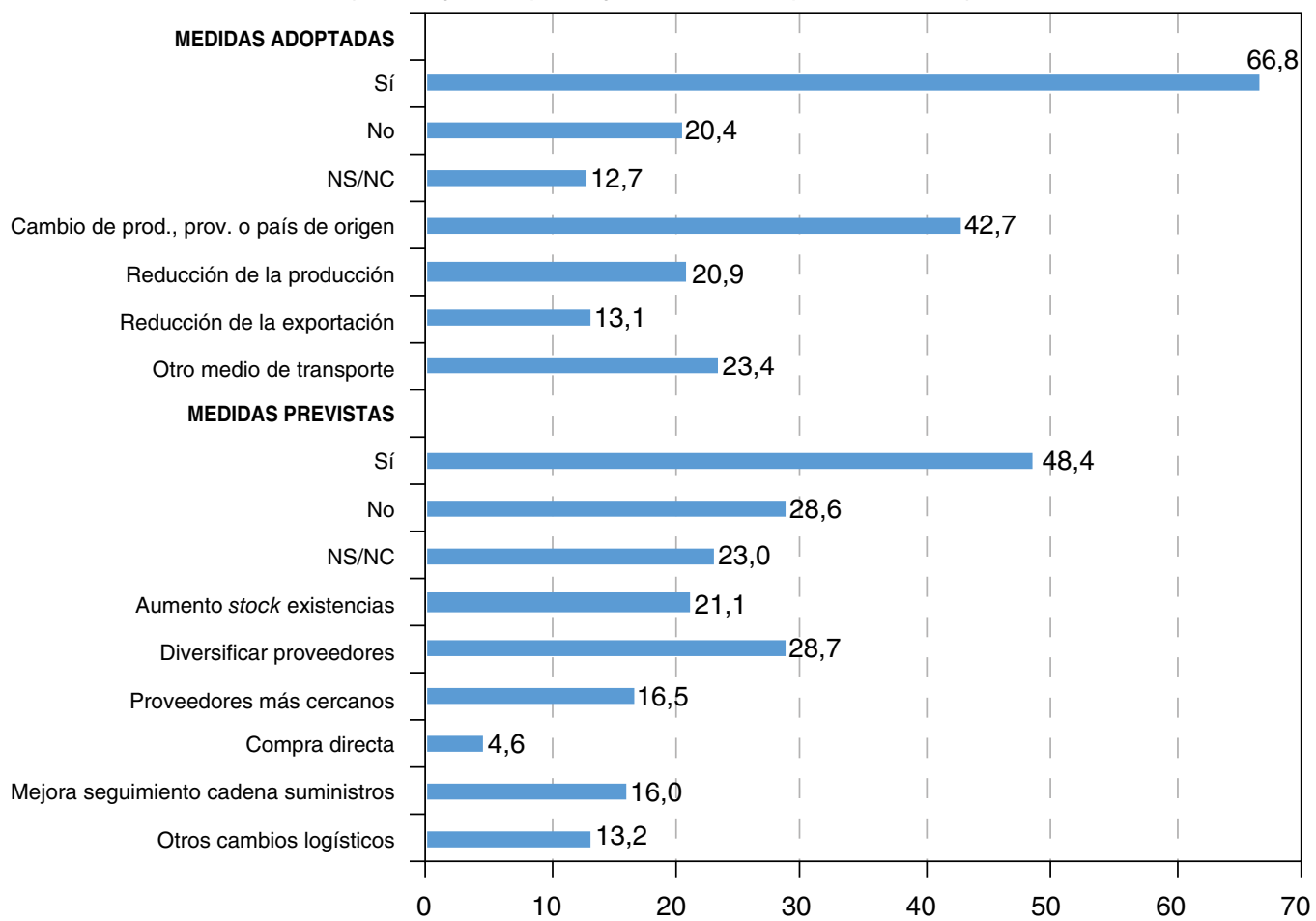

Fuente: Encuesta de Coyuntura de la Exportación, IV trimestre de 2021. 
consumo duradero. En estos sectores, los exportadores que han adoptado alguna medida ante las dificultades de abastecimiento que están sufriendo atribuyen un peso a la reducción de la producción, como medida compensatoria, del 31,5\% en el sector del automóvil y del $30,7 \%$ en el caso del sector de bienes de consumo duradero.

Es destacable que, en todos los sectores, la importancia relativa que atribuyen las empresas que han adoptado medidas frente a los problemas de aprovisionamiento a la reducción de la producción supera a la atribuida a la reducción de las exportaciones. Esta diferencia podría ser indicativa de un elevado nivel de concienciación de los exportadores regulares sobre la necesidad de mantenener sus mercados exteriores, a pesar de que es probable que, al menos en algunos casos, los problemas logísticos también estén dificultando o encareciendo los flujos de exportación.

Por otro lado, la idea de que los riesgos asociados al aprovisionamiento han adquirido mayor relevancia y de que deben ser atendidos en mayor medida parece estarse consolidando entre los exportadores regulares. Como estrategia de adaptación al nuevo entorno, las empresas que declaran tener prevista la adopción de nuevas medidas optan principalmente por la diversificación de proveedores, el acortamiento de sus cadenas de suministro y el aumento de stocks. Estas medidas, no exentas de coste, son consistentes con el objetivo de reducir riesgos de suministro en el futuro. Así, el $48,4 \%$ de las empresas que exportan regularmente prevé adoptar nuevas medidas cuando se normalice la situación (Gráfico 7 y Cuadro 3). Entre las medidas previstas por este colectivo de empresas destacan la diversificación de proveedores (con un coeficiente de importancia relativa del $28,7 \%$ ), el aumento del stock de existencias $(21,1 \%)$, acudir a proveedores más cercanos $(16,5 \%)$, la mejora del seguimiento de la cadena de aprovisionamiento (16,0\%) y otros cambios logísticos (13,2\%). El recurso a compras directas, prescindiendo de intermediarios, recibe una valoración relativa de solo un $4,6 \%$.

La diversificación de proveedores y el aumento de stocks son las medidas a las que las empresas asignan una mayor valoración relativa en cada uno de los sectores de $D$

CUADRO 2

MEDIDAS ADOPTADAS POR LOS EXPORTADORES REGULARES

(Porcentaje de empresas y coeficiente de importancia relativa)

\begin{tabular}{|l|c|c|c|c|c|c|c|}
\hline & \multicolumn{2}{|c|}{ Ha adoptado medidas } & \multicolumn{5}{c|}{ Medidas adoptadas } \\
\cline { 2 - 8 } & Sectores & No & NS/NC & $\begin{array}{c}\text { Cambio de producto, } \\
\text { proveedor o país de } \\
\text { origen }\end{array}$ & $\begin{array}{c}\text { Reducción } \\
\text { de la } \\
\text { producción }\end{array}$ & $\begin{array}{c}\text { Reducción } \\
\text { de la } \\
\text { exportación }\end{array}$ & $\begin{array}{c}\text { Otro medio } \\
\text { de } \\
\text { transporte }\end{array}$ \\
\hline Alimentación, bebidas y tabaco & 59 & 26 & 15 & 43,9 & 23,3 & 16,6 & 16,1 \\
Productos energéticos & 50,2 & 49,5 & 0,3 & 59,5 & 20,1 & 0,7 & 19,7 \\
Materias primas & 66,8 & 30,6 & 2,6 & 58 & 24,6 & 15,3 & 2,2 \\
Semimanufacturas no químicas & 70,7 & 16,5 & 12,8 & 47 & 22,5 & 14,3 & 16,2 \\
Productos químicos & 80,8 & 6,6 & 12,6 & 44,7 & 20,8 & 12,3 & 22,1 \\
Bienes de equipo & 64,8 & 20,8 & 14,4 & 43 & 17,8 & 11,7 & 27,6 \\
Sector del automóvil & 66,9 & 19,3 & 13,8 & 26,2 & 31,5 & 21 & 21,2 \\
Bienes de consumo duradero & 49,6 & 24 & 26,5 & 28,7 & 30,7 & 6,2 & 34,5 \\
Manufacturas de consumo & 71,6 & 18,2 & 10,2 & 43,3 & 10,9 & 10,2 & 35,5 \\
Total & $\mathbf{6 6 , 8}$ & $\mathbf{2 0 , 4}$ & $\mathbf{1 2 , 7}$ & $\mathbf{4 2 , 7}$ & $\mathbf{2 0 , 9}$ & $\mathbf{1 3 , 1}$ & $\mathbf{2 3 , 4}$ \\
\hline
\end{tabular}


exportación, excluido el de materias primas. Posiblemente por la especificidad de este tipo de productos y por el elevado coste de almacenamiento que, en algunos casos, pueden presentar, los exportadores regulares de materias primas otorgan prioridad a otro tipo de medidas, como la compra directa (22,6\%), la mejora del seguimiento de la cadena de aprovisionamiento $(22,6 \%)$ y otros cambios logísticos $(22,6 \%)$.

\section{Conclusiones}

El sector exportador está siendo clave para atenuar el impacto económico de la pandemia e impulsar una recuperación rápida y sostenible. Su favorable inserción en cadenas globales de valor es uno de los elementos que explica su elevada capacidad de contribución al crecimiento económico. Sin embargo, la inserción en cadenas globales de valor también supone una elevada exposición de los exportadores a los problemas de suministro que están observándose en la actualidad.
El análisis de las opiniones empresariales recogidas en la Encuesta de Coyuntura de la Exportación revela que los exportadores regulares de bienes son conscientes de estos riesgos. Desde 2021, y en especial desde mediados del año, las empresas que exportan bienes de forma regular están afrontando problemas de abastecimiento, derivados de los mercados internacionales, que limitan su capacidad de producir o exportar. Estos problemas se concretan en retrasos en la entrega de mercancías, incremento de los costes de transporte o de los propios productos o, más raramente, dificultades para encontrar el producto deseado.

Para hacer frente a estos problemas, los exportadores han recurrido preferentemente a cambios en los proveedores o en los productos que precisan y al recurso a otros mercados de origen, procurando en todo caso mantener sus mercados exteriores. En una perspectiva temporal más amplia, las empresas exportadoras están adaptando sus estrategias a lo que parecen percibir como una situación en la que $\triangleright$

\section{CUADRO 3}

PREVISIÓN DE ADOPCIÓN DE NUEVAS MEDIDAS, CUANDO SE NORMALICE LA SITUACIÓN (Porcentaje de empresas y coeficiente de importancia relativa)

\begin{tabular}{|c|c|c|c|c|c|c|c|c|c|}
\hline \multirow[b]{2}{*}{ Sectores } & \multicolumn{3}{|c|}{$\begin{array}{l}\text { Prevé adoptar } \\
\text { nuevas medidas }\end{array}$} & \multicolumn{6}{|c|}{ Medidas que prevé adoptar } \\
\hline & Sí & No & $\begin{array}{l}\text { NS/ } \\
\text { NC }\end{array}$ & $\begin{array}{l}\text { Aumento } \\
\text { stock } \\
\text { existencias }\end{array}$ & $\begin{array}{l}\text { Diversificar } \\
\text { proveedores }\end{array}$ & $\begin{array}{l}\text { Proveedores } \\
\text { más } \\
\text { cercanos }\end{array}$ & $\begin{array}{l}\text { Compra } \\
\text { directa }\end{array}$ & $\begin{array}{c}\text { Mejora } \\
\text { seguimiento } \\
\text { cadena } \\
\text { suministros }\end{array}$ & $\begin{array}{c}\text { Otros } \\
\text { cambios } \\
\text { logísticos }\end{array}$ \\
\hline Alimentación, bebidas y tabaco & 51,5 & 26,6 & 21,9 & 19,3 & 29,1 & 14,2 & 8,2 & 17,9 & 11,3 \\
\hline Productos energéticos & 33,5 & 65,8 & 0,7 & 33,7 & 33,1 & 0,0 & 0,0 & 32,6 & 0,6 \\
\hline Materias primas & 29,8 & 29,3 & 40,9 & 7,0 & 12,5 & 12,8 & 22,6 & 22,6 & 22,6 \\
\hline Semimanufacturas no químicas & 49,4 & 23,3 & 27,3 & 21,1 & 28,8 & 12,9 & 5,7 & 13,5 & 18,0 \\
\hline Productos químicos & 52,4 & 16,5 & 31,1 & 20,3 & 29,8 & 16,1 & 5,1 & 15,9 & 12,8 \\
\hline Bienes de equipo & 48,0 & 27,9 & 24,1 & 21,8 & 31,6 & 15,5 & 2,4 & 14,1 & 14,6 \\
\hline Sector del automóvil & 34,7 & 44,2 & 21,1 & 19,2 & 27,1 & 24,2 & 1,5 & 17,6 & 10,3 \\
\hline Bienes de consumo duradero & 59,6 & 6,4 & 34,0 & 30,2 & 22,8 & 9,4 & 3,6 & 18,3 & 15,6 \\
\hline Manufacturas de consumo & 62,6 & 19,8 & 17,6 & 20,9 & 23,1 & 24,3 & 4,6 & 14,1 & 13,0 \\
\hline Total & 48,4 & 28,6 & 23,0 & 21,1 & 28,7 & 16,5 & 4,6 & 16,0 & 13,2 \\
\hline
\end{tabular}

Fuente: Encuesta de Coyuntura de la Exportación, IV trimestre de 2021. 
los riesgos de abastecimiento pueden continuar siendo relevantes.

La adaptación de estrategias supone adoptar medidas de diversificación o atenuación del riesgo, como la diversificación de proveedores - la compra a proveedores más próximos, acortando la cadena de valor, así como incurrir en mayores costes de aseguramiento frente a posibles problemas de suministro, mediante el incremento de los stocks de existencias. La mejora del seguimiento de las cadenas de suministro y la mayor atención a la logística son otros ámbitos de actuación hacia los que se orienta la estrategia empresarial.

Las políticas de apoyo a la internacionalización deben acompañar a las empresas en este esfuerzo, adaptando sus instrumentos a las nuevas necesidades percibidas. Es conveniente que las políticas de apoyo a la internacionalización refuercen los instrumentos que faciliten a las empresas la formación y el soporte que puedan requerir para mejorar su control sobre la cadena de suministros, así como las herramientas que les permitan identificar proveedores alternativos de los insumos que precisan y acceder a servicios logísticos competitivos y fiables.

En este marco, cobra especial importancia, tanto para las empresas exportadoras como para las autoridades públicas, la identificación de productos importados que puedan considerarse críticos para la exportación, en especial si existe dependencia de países no comunitarios en los que pueda concentrarse un mayor riesgo geopolítico. El análisis de la dependencia geográfica de las importaciones es un primer paso para esta identificación, que debe completarse con un estudio del valor estratégico de estos inputs y de las potenciales fuentes de suministro alternativas, común para toda la economía, y con un análisis específico de la relevancia de estos inputs para el sector exportador. Como se deduce del análisis preliminar realizado en estas páginas, es probable que al menos una parte importante de los suministros estratégicos en los que existe dependencia sean comunes para toda la Unión Europea, por lo que, en general, será deseable y más eficaz plantear medidas coordinadas a nivel comunitario.

\section{Bibliografía}

Comisión Europea. (2021). Commission Staff Working Document, Strategic Dependencies and Capacities. SWD 2021/352 final. https://ec.europa.eu/info/sites/default/files/strategic-dependencies-capacities.pdf

Ministerio de Industria, Comercio y Turismo. (2021a). El Plan de Acción para la Internacionalización de la Economía Española 2021-2022. https://comercio.gob.es/es-es/estrategia_internacionalizacion/Paginas/plan-accion-2021-2022.aspx

Ministerio de Industria, Comercio y Turismo. (2021b). Encuesta de Coyuntura de la Exportación. https://comercio.gob.es/ImportacionExportacion/Informes_Estadisticas/Paginas/Encuesta-de-coyuntura.aspx

OCDE. Statistics on Trade in Value Added. https:// doi.org/10.1787/36ad4f20-en

Subdirección General de Estrategia de Internacionalización. (2021). El Plan de Acción para la Internacionalización de la Economía Española 2021-2022. Boletín Económico de ICE, (3138). https://doi.org/10.32796/bice.2021.3138.7283

Subdirección General de Estudios y Evaluación de Instrumentos de Política Comercial. (2021). Recuperación económica y problemas de abastecimiento: el papel de los fletes. Boletín Económico de ICE, (3141). https://doi.org/10.32796/bice. 2021.3141 .7326

Web DataComex de la Secretaría de Estado de Comercio. https://datacomex.comercio.es/ 